\title{
EDITORIAL
}

\section{LA FORMACIÓN EN ESPAÑA DE MÉDICOS ESPECIALISTAS EN MEDICINA PREVENTIVA Y SALUD PÚBLICA}

\author{
Joan R Villalbí, Gloria Pérez-Albarracín, Miguel Delgado-Rodríguez y el Grupo de reflexión \\ sobre el futuro de la especialidad de Medicina Preventiva y Salud Pública.
}

El grupo de reflexión está formado por miembros de la Comisión Nacional de la Especialidad de Medicina Preventiva y Salud Pública, e incluye a Ana Almaraz, Miguel Delgado-Rodríguez, Joaquín Fernández-Crehuet, F Javier García-León, Jesús Lozano, Gloria Pérez-Albarracín, Andreu Segura, Joan R Villalbí y Vicente Zanón, que son los miembros senior de la comisión durante el periodo 2010-11 (salvo los representantes de los residentes), y que han compartido perspectivas y experiencia sobre este aspecto tras las reuniones de la Comisión.

El modelo moderno de formación médica especializada en España se definió en $1984^{1}$ y se basa en el programa de Médicos Internos Residentes (MIR). En 1999 se produjo el acceso al título de especialista de buena parte de la gran bolsa de médicos que trabajaban en el sector sin tener título oficial de especialista (denominados médicos especialistas sin título oficial (MESTOS) mediante una convocatoria excepcional del Consejo Nacional de Especialidades Médicas ${ }^{2}$. Desde entonces, la oferta de formación mediante la convocatoria MIR es el esquema predominante, aunque persiste la vía excepcional y sea factible la homologación de títulos de especialista obtenidos en el extranjero. Este modelo se ha perfeccionado en los últimos tiempos ${ }^{3}$, destacando la aprobación en 2005 del programa de la especialidad de Medicina Preventiva y Salud Pública(MPySP) ${ }^{4,5}$, y avanzando en una mayor definición del funcionamiento de las unidades docentes, de las comisiones de docencia, y de los tutores ${ }^{6,7}$.
Al redactar este documento nos proponemos proporcionar algunos elementos de información sobre la oferta de formación médica especializada en MPySP. La información descriptiva se ha extraído de las últimas convocatorias publicadas en el Boletín Oficial del Estado (BOE), correspondientes al periodo 2007-10. El esbozo de elementos para la evaluación procede de reflexiones compartidas por los miembros de la Comisión Nacional de la Especialidad (CNE) a lo largo del período 2010-11, los cuales son especialistas. Independientemente de que puedan darse progresos en la sistematización de la formación especializada de otras profesiones implicadas en la salud pública, algo muy deseable ${ }^{8}$, nos concentramos aquí en la situación de esta especialidad médica.

Oferta de plazas. La oferta de plazas de formación de especialista en MP y SP se ha estabilizado en España, y actualmente es de unas 80 plazas al año, cifra algo superior al $1 \%$ de la oferta total de formación especiali- 
zada, que supera las 7.000 plazas. Es el doble que las 40 de hace 15 años, pero ha dejado de crecer y es probable que ya no varíe mucho en el futuro. La distribución geográfica por Comunidades Autónomas (CCAA) de la oferta de plazas viene determinada por la distribución de las unidades acreditadas, con pequeñas oscilaciones (anexo 1). La acreditación se realiza de manera cada vez más formalizada, de acuerdo con instrumentos normalizados ${ }^{9}$. Tan solo tres CCAA (Asturias, Baleares y La Rioja) carecen de unidades acreditadas de formación en 2010, y todas ellas están entre las comunidades con menor población. La oferta se concentra en las cuatro CCAA más pobladas, Madrid (25 en 2010), seguida de Andalucía (20 en 2010) y Cataluña (13 en 2010). Sólo la Comunidad Valenciana tuvo 10 y las demás están por debajo, siendo Cantabria y Extremadura las únicas que tienen una sola plaza. No siempre se convocan todas las plazas acreditadas, hecho que es más visible en algunas de las CCAA con mayor oferta. Esto parece ser debido a que en algunas se acreditaron más plazas de las necesarias aspirando a introducir así elementos de competitividad entre unidades que pudieran servir de estímulo a la calidad. En la práctica no parece que este mecanismo haya sido realmente utilizado.

En las CCAA con menor oferta, se concentra en una única unidad, que a veces opera en diversos centros (sobre todo hospitales) o áreas. En Andalucía o Aragón hay una gran unidad administrativa que centraliza algunos aspectos, aunque las plazas están en realidad asignadas a centros hospitalarios y otros dispositivos docentes donde se realiza el grueso de la formación. En el caso de Madrid hay dos, debido a que el Instituto de Salud Carlos III, que depende de la administración central, mantiene una propia). En otras comunidades, como Cataluña o la Comunidad Valenciana, hay diferentes unidades docentes con identidad propia.
Tras el examen, los futuros residentes eligen por orden de puntuación su plaza entre las ofertadas vacantes. Aunque en 2009 en alguna unidad de ámbito autonómico sólo se daba opción a los nuevos residentes a optar por la unidad central, y luego se les asignaba a los diversos centros, lo que resultó en más vacantes y algunas renuncias, por lo que no parece probable que se mantenga en años sucesivos.Lo habitual es que el residente elija plaza directamente en el centro docente.

La especialidad de MPySP requiere una formación teórica inicial que se proporciona mediante un programa de maestría en salud pública al que se consagra el primer año de formación. En 2009 había cinco programas de maestría utilizados por las unidades. Algunos están vinculados a universidades, y están en curso de acreditación nuevos programas de maestría. El más antiguo y con mayor número de participantes es el de la Escuela Nacional de Sanidad en Madrid, adscrita al Instituto de Salud Carlos III de la administración central. De los otros, destacan cuantitativamente los de Granada y Barcelona. El de Valencia se realiza cada dos años, por lo que los residentes suelen ir a la ENS en los años en que no se realiza. En algunos de estos programas, la realización de un trabajo de investigación o tesina es un requisito crucial para su superación, que comporta un esfuerzo notable y el aprendizaje de habilidades con un fuerte valor añadido.

Estudios disponibles y la información sobre la adjudicación de plazas de formación médica especializada en las últimas convocatorias permiten formular algunos juicios globales $^{10-12}$. La especialidad de MPySP se puede considerar básicamente como no hospitalaria, situación que comparte con la de Medicina Familiar y Comunitaria y la de Medicina del Trabajo, especialidades en las que los residentes cobran mientras se forman (en contraste con las denominadas de escuela profesional, donde los futuros especialistas han de financiar su formación, como 
Medicina Legal o Medicina del Deporte). En el contexto general ha habido cambios que afectan tanto al conjunto de la convocatoria MIR como a la especialidad de MPySP, de los que destaca el crecimiento en la oferta de plazas (de 5.242 en 2001 a 6.881 en 2010) y su atracción sobre profesionales extranjeros, procedentes o no de la Unión Europea y formados o no en España. Entre 2001 y 2009 los profesionales extranjeros pasaron de ocupar 126 plazas asignadas (el 2,40\%), a 1.934 (el 28,84\%). Estos cambios tienen una poderosa influencia en la asignación y ocupación de plazas. Además, hay tres elementos relevantes que se desprenden de los datos existentes: las plazas que quedan sin asignar, las preferencias de los que superan el examen MIR y la recirculación de residentes.

Plazas sin asignar. Apenas quedan plazas vacantes en las convocatorias MIR. De las plazas ofertadas para residentes en las diversas especialidades que no son de escuela profesional no suelen quedar plazas sin asignar (salvo en los últimos años en medicina familiar y comunitaria). Sin embargo, algunas plazas asignadas no son finalmente ocupadas.

Preferencia y ranking. Se aprecia una gran dispersión en la elección. Por una parte, la especialidad de MPySP es elegida por algunas personas muy vocacionales con buenos resultados en el examen MIR, pero algunas de sus plazas son de las últimas que se eligen. La mediana del ranking de quien la elige es comparable al de diversas especialidades hospitalarias (radiología, alergología, neumología, reumatología, geriatría, microbiología...) y más favorable que el de medicina familiar y comunitaria, pero esto se acompaña de una desviación típica muy elevada.

Recirculación de residentes entre especialidades. La formación en la especialidad de MPySP no parece especialmente afectada por la recirculación de profesionales (los que abandonan su residencia sin haberla termi- nado para volver a presentarse al examen MIR). Este fenómeno, que afecta mucho a las especialidades de escuela profesional, bastante a especialidades de laboratorio y con cierta frecuencia a la especialidad de Medicina Familiar y Comunitaria, no parece manifestarse especialmente en MPySP. En cambio, cada año algunos especialistas en Medicina Familiar y Comunitaria vuelven a presentarse al examen MIR y eligen nueva formación en MPySP.

¿Cuales son nuestras necesidades de información para el futuro? Se dispone de un esquema básico de evaluación de las unidades docentes, a partir de los requisitos para su acreditación ${ }^{13}$ : aunque el manual aún sea el anterior a la aprobación del actual programa de la especialidad y esté pendiente de actualizarse, contiene elementos de interés ${ }^{14}$. Para avanzar hacia un esquema de evaluación, se pueden valorar de manera clásica aspectos de evaluación del proceso y de evaluación de los resultados.

Respecto a los indicadores de proceso se puede evaluar el grado de conformidad de la docencia y de las actividades realizadas con las previstas, y la existencia de mecanismos para medir la calidad. Algunas especialidades han desarrollado instrumentos que van en esta dirección: representan un paso adelante respecto al procedimiento de acreditación inicial de las unidades ${ }^{15}$. Esporádicamente algún programa de maestría también ha intentado evaluarse de forma integral y objetiva, pero no sistemáticamente ${ }^{16}$. Estos instrumentos suelen ser cualitativos y dicotómicos, pero nada impediría que evolucionaran hacia instrumentos que proporcionasen una puntuación numérica u ordinal, más orientada a estimular las mejoras mediante procesos de benchmarking.

Respecto a los indicadores de resultado, se pueden obtener mediante instrumentos del tipo encuesta o prueba, o mediante la valoración de la trayectoria de los residentes, incluyendo la que siguen tras culminar su 
formación como especialistas. La obtención de algunos de estos componentes requiere un esfuerzo: aunque están disponibles en algunas unidades, su uso no se encuentra generalizado. Contrariamente, otros podrían obtenerse con un esfuerzo relativamente modesto de recogida de información. Su uso debería ser sistemático y los indicadores deberían ofrecerse acumulando información de varios años, pues de otro modo, en la mayoría de las unidades los datos más cuantitativos se basarían en pocas observaciones sujetas a gran variabilidad.

En definitiva, yendo más allá de la gestión rigurosa del día a día, para el futuro creemos que hay dos retos principales que además están relacionados: 1) consolidar y prestigiar la especialidad entre los licenciados en medicina y el sistema nacional de salud y 2) elaborar criterios para poder evaluar la formación ofrecida y utilizar posteriormente sus frutos para mejorar la formación. Esto ha de permitir orientar la demanda de los médicos que buscan especializarse. En este sentido, conocer mejor la estructura del sistema de formación es un primer paso necesario.

La evaluación sistemática de la calidad de las actividades de formación de especialistas está aún poco implantada en nuestro país. Por ello, una aproximación inicial a su extensión ha de ser prudente. El objetivo es usar la evaluación para mejorar la formación, no para elaborar cuadros de honor. Esto sólo será posible con un proceso de consenso en el seno de la CNE y un trabajo participativo con las unidades docentes previo a la implantación de la evaluación.

El acceso al sistema MIR en España se basa en los resultados de un examen centralizado que mide exclusivamente los conocimientos. En países como los EEUU los sistemas de selección incorporan otros componentes además del examen, buscando evaluar las actitudes y habilidades de los candidatos $1^{17}$. La evaluación de la formación adquirida por los residentes apenas se abor- da, aunque existe un cierto consenso respecto a su calidad general. De hecho, en el campo de la salud pública tan sólo los médicos disponen de una formación reglada generalizada, pues para los profesionales del sector con otras titulaciones de base (como veterinaria o farmacia, mayoritarios en las dependencias de protección de la salud) no está desarrollada más allá de la oferta de maestrías de salud pública, ni se exige habitualmente para su acceso a los lugares de trabajo. Esto sucede también en otros segmentos del sistema sanitario, como la atención primaria, en la que la formación ofrecida a los residentes en medicina familiar y comunitaria no tiene equivalente en otras profesiones. Sin embargo, plantea la existencia de carencias de otros profesionales que ejercen en la salud pública sin haber recibido formación específica, algo que debería poder corregirse en el futuro tal como preveía la ley de profesiones sanitarias ${ }^{3,8,18}$. La calidad de la formación especializada de los médicos especialistas en MPySP debería ser un estímulo para desarrollar programas de formación especializada en las otras profesiones que tienen un papel en este campo, garantizando sus competencias específicas ${ }^{19}$.

\section{BIBLIOGRAFÍA}

1. Boletín Oficial del Estado. Real Decreto 127/1984, de 11 de enero, por el que se regula la Formación Médica Especializada y la obtención del Título de Médico Especialista.BOE número 103 de 30/4/1984.

2. Boletín Oficial del Estado. Real Decreto 1497/1999, de 24 de septiembre, por el que se regula un procedimiento excepcional de acceso al título de Médico Especialista.BOE número 230 de 25/9/1999.

3. Boletín Oficial del Estado. Ley 44/2003, de 21 de noviembre, de ordenación de las profesiones sanitarias. BOE número 280 de 22/11/2003.

4. Gómez-López L, Sangrador-Arenas L. Programa docente de la especialidad de medicina preventiva y salud pública. Rev San Hig Pub 1995; 69: 1-4.

5. Boletín Oficial del Estado. SCO/1980/2005, de 6 de junio, por la que se aprueba y publica el programa formativo de la especialidad de Medicina Preventiva y Salud Pública.BOE número 152 27/06/2005. 
6. Boletín Oficial del Estado.Real Decreto 183/2008, de 8 de febrero, por el que se determinan y clasifican las especialidades en Ciencias de la Salud y se desarrollan determinados aspectos del sistema de formación sanitaria especializada.BOE número 45 de 21/2/2008.

7. Boletín Oficial del Estado. SCO/581/2008, de 22 de febrero, por la que se publica el Acuerdo de la Comisión de Recursos Humanos del Sistema Nacional de Salud, por el que se fijan criterios generales relativos a la composición y funciones de las comisiones de docencia, a la figura del jefe de estudios de formación especializada y al nombramiento del tutor.BOE número 56 de 5/3/2008.

8. Benavides FG. Acerca de la formación de los profesionales de salud pública, algunos avances y muchos retos. Informe SESPAS 2010. Gac Sani. 2010; 24 (Supl 1): $90-5$

9. Auditoria docente de la especialidad de medicina preventiva y salud pública. Requisitos mínimos para la acreditación de la Comisión Nacional de la Especialidad. Madrid: Ministerio de Sanidad y Consumo, sin fecha. Disponible en: http://www.msc.es/organizacion/sns/planCalidadSNS/pdf/excelencia/cuestionario/M_Preventiva_y_Salud_Publica.pdf

10. Gonzalez López-Valcárcel B, Barber Pérez B. Programa MIR como innovación y como mecanismo de asignación de recursos humanos. En: Meneu R, Ortún $\mathrm{V}$, Rodríguez-Artalejo F (Eds) Innovaciones en gestión clínica y sanitaria. Barcelona: Masson, 2005; 101-26.

11. Barber Pérez P, González Gómez-Valcárcel B. Oferta y necesidad de médicos especialistas en España (2008-2025). Diciembre 2008. Las Palmas de Gran Canaria: Universidad de las Palmas; 2009 (disponible en :

http://www.msps.es/profesionales/formacion/docs/nec esidadesEspecialistas2008_2025.pdf).

12. Romeo-Ladrero JM. Entrelazados II (disponible en http://mirentrelazados.blogspot.com/).

13. Requisitos generales de acreditación de las unidades docentes para la formación de especialistas en medicina preventiva y salud pública. Madrid: Ministerio de Sanidad y Consumo y Ministerio de Educación y Ciencia; 2006. Disponible en: http://www.msc.es/profesionales/formacion/docs/medicinaPreventiva2010.pdf.

14. Auditoria docente de la especialidad de Medicina Preventiva y Salud Pública. Requisitos mínimos para la acreditación de la Comisión Nacional de la Especialidad. Disponible en:

http://www.msc.es/organizacion/sns/planCalidadSNS/pdf/excelencia/cuestionario/M Preventiva y _Salud_Publica.pdf
15. Terol García E, Palanca Sánchez I (Coord) Manual de auditoria docente. Especialidad de Medicina Familiar y Comunitaria. Madrid: Ministerio de Sanidad y Consumo; 2006. Disponible en : http://www.msc.es/organizacion/sns/planCalidadSNS/pdf/excelencia/cuestionario/Medicina_Familiar_y_Comunitaria.pdf

16. Segura A, Villalbí JR, Costa J, Canela J. Formació en salut pública i administració sanitària. En: Llibre de ponències. Lleida: XV Congrés de metges i biòlegs de llengua catalana; 1996; 281-90.

17. Gual A, Pardell H (Coords). El médico del futuro. Barcelona: Fundación Educación Médica; 2009

18. Institut d'Estudis de la Salut. Els recursos humans en salut pública. Competències professionals i formació necessària. Barcelona: Departament de Salut de la Generalitat de Catalunya, 2010. Disponible en: http://www.gencat.cat/salut/ies/html/ca/dir2631/llibreasp.pdf.

19. Benavides FG, Moya C, Segura A, de la Puente ML, Porta M, Amela C y el Grupo de Trabajo sobre Competencias Profesionales en Salud Pública. Las competencias profesionales en salud pública. Gac Sanit. 2006; 20:239-43. 


\section{Anexo 1}

Distribución por Comunidades Autónomas de la oferta de formación en medicina preventiva y salud pública. España, 2007-2010

\begin{tabular}{|l|c|c|c|c|c|}
\hline Comunidad Autónoma & $\begin{array}{c}\text { Oferta } \\
2007\end{array}$ & $\begin{array}{c}\text { Oferta } \\
2008\end{array}$ & $\begin{array}{c}\text { Oferta } \\
2009\end{array}$ & $\begin{array}{c}\text { Oferta } \\
2010\end{array}$ & $\begin{array}{c}\text { Plazas } \\
\text { acreditadas } \\
2010\end{array}$ \\
\hline Andalucía & 10 & 12 & 13 & 13 & 20 \\
\hline Aragón & 6 & 6 & 6 & 4 & 6 \\
\hline Canarias & 2 & 2 & 2 & 2 & 2 \\
\hline Cantabria & 1 & 1 & 1 & 1 & 1 \\
\hline Castilla-La Mancha & 6 & 6 & 6 & 6 & 6 \\
\hline Castilla-León & 5 & 5 & 5 & 5 & 5 \\
\hline Cataluña & 11 & 11 & 11 & 11 & 13 \\
\hline Extremadura & - & - & 1 & 1 & 1 \\
\hline Galicia & 3 & 3 & 3 & 4 & 5 \\
\hline Madrid & 22 & 24 & 23 & 21 & 25 \\
\hline Murcia & 4 & 3 & 3 & 2 & 4 \\
\hline Navarra & 2 & 2 & 2 & 1 & 2 \\
\hline País Vasco & 2 & 2 & 2 & 2 & 2 \\
\hline Comunidad Valenciana & 6 & 10 & 9 & 9 & 10 \\
\hline Total & 80 & 87 & 87 & 82 & 102 \\
\hline
\end{tabular}

El número de plazas acreditadas se extrae de la reunión de Unidades Docentes de marzo 2011. No hay unidades acreditadas en Asturias, Baleares y la Rioja en 2010. 